\title{
Capillary waveguide biosensor
}

\author{
Hanna Stawska • Mariusz Śliwa • Marcin Kochanowicz • \\ Jacek Żmojda • Karol Czajkowski • Dominik Dorosz • \\ Elżbieta Bereś-Pawlik
}

Received: 3 March 2014 / Accepted: 20 August 2014 / Published online: 29 August 2014

(C) The Author(s) 2014. This article is published with open access at Springerlink.com

\begin{abstract}
In this paper a new construction of the capillary waveguide biosensor is presented. The sensor is applied for fluorescence intensity measurements and the detection of dye concentration. We present the results of the measurements of different dye concentration to standardize this sensor as a biosensor for potential applications in the detection of fluorophores nicotinamide adenine dinucleotide and flavins which can be considered as metabolomic markers of cancer.
\end{abstract}

Keywords Biosensor - Capillary waveguide · Fluorescence spectroscopy · Evanescent waveguide $\cdot$ Cancer detection

\section{Introduction}

Fluorescence spectroscopy is a widely used method for cancer detection. Nowadays a lot of effort is made in order to develop novel fluorescence techniques and improve existing ones, which can be used with newly developed fluorescent probes for specific cancer diagnosing. For example, recently, native fluorescence characteristics of human urine samples were examined using excitation-emission matrices over a range of excitation and emission wavelengths, and emission spectra at $405 \mathrm{~nm}$ excitation Ramu et al. (2013). It was shown that fluorophores nicotinamide adenine dinucleotide (NADH) and flavins can be considered as metabolomic markers of cancer Ramu et al. (2013). Simultaneously, researchers and physicians need a simple and fast method to obtain data instantaneously for the detection of specific properties of a sample. Therefore they search for biosensors which allow for the examination

H. Stawska $(\varangle) \cdot$ M. Śliwa · E. Bereś-Pawlik

Telecommunications and Teleinformatics Department, Wrocław University of Technology,

Wybrzeże Wyspiańskiego 27, 50-370 Wrocław, Poland

e-mail: hanna.stawska@pwr.wroc.pl

M. Kochanowicz · J. Żmojda · K. Czajkowski · D. Dorosz

Department of Power Engineering, Photonics and Lighting Technology, Faculty of Electrical Engineering, Białystok University of Technology, Wiejska 45D, 15-351 Białystok, Poland 
of a broad spectrum of substances (food, blood, urine and serum). Capillary waveguides could serve as such biosensors due to their capabilities: they could simultaneously serve as optical and sampling cells, which simplifies the measurements setup, and they also could be easily coupled to light sources and detectors Keller et al. (2007). Most often capillary waveguides are applied in two ways: as a core propagating liquid waveguide or as a wall propagating evanescent waveguide. The way the sensor works depends upon its construction and also the refractive index (RI) of the medium filling the capillary core. In the core propagating waveguides, the core is filled with a substance with a higher refractive index than the refractive indexes of the wall and light is coupled into the core, not into the wall made from BK7 glass. This construction allows light to propagate through the waveguide by the total internal refraction. This type of a waveguide biosensor provides increased sensitivity because light travels directly through the solution filled core over a long path length. In the case of the capillary evanescent waveguide, the fluorescence is excited in the vicinity of the inner surface of the capillary where the evanescent field is at a maximum. In this approach we propose a new capillary waveguide which consists of several capillaries and uses both methods to excite the fluorescence signal: direct as a liquid core waveguide and indirect as an evanescent field sensor.

\section{Capillary biosensor: structure and properties}

The typical construction of waveguide biosensors assumes the application of only one capillary. In our approach we would like to enhance the sensitivity of the biosensor so we simultaneously used several capillaries. In Fig. 1 the structure of the proposed capillary waveguide biosensor is presented. The capillary waveguide biosensor consists of 37 capillaries made from BK7 glass which are arranged in a hexagonal lattice. The biosensor has length $\mathrm{L}=1 \mathrm{~cm}$. These capillaries have inner radius $\mathrm{R}_{\mathrm{i}}=163 \mu \mathrm{m}$ and outer radius $\mathrm{R}_{\mathrm{o}}=271 \mu \mathrm{m}$. The central capillary has inner radius $R_{i}=206 \mu \mathrm{m}$ and outer radius $R_{o}=290 \mu \mathrm{m}$. The total volume of this capillary biosensor is equal to about $\mathrm{V}=43.31 \mu \mathrm{L}$. By appropriately selecting the dimensions of the capillaries and due to the phenomenon of viscosity there is no need for the application of additional components to protect the solution from leaking. In our case the idea of the sensor is the result of the application of the preform which was prepared during the manufacturing of a previously elaborated double clad hollow core fiber

Fig. 1 Cross section of the examined capillary biosensor

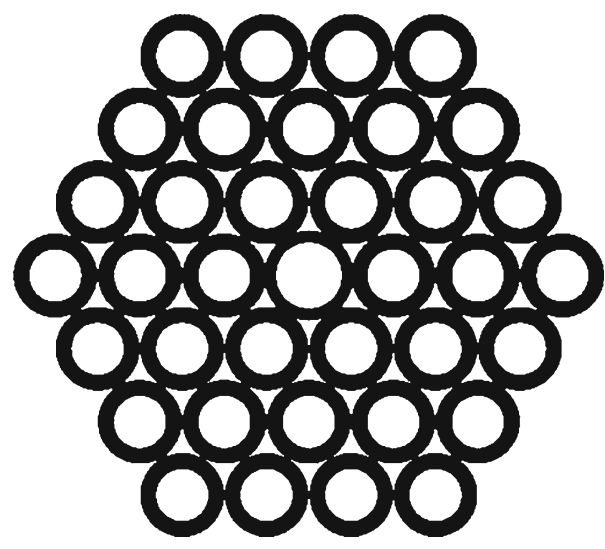


for two photon endoscopy. The preform which remained unused during the production of the fiber was cut into smaller pieces and used as a biosensor. Therefore, the inner capillary inside the biosensor has different dimensions in comparison to the other capillaries.

Assuming that the biosensor is illuminated by a collimated beam, the light is propagated both in the glass and in the solution. In such case the fluorescence is excited not only directly in the solution but also at the boundary of the glass and the solution by the evanescent field present at the interface of the total internal reflection (TIR). Due to TIR, which occurs at an optical interface when the angle of incidence of light propagating within the waveguide is greater than $\phi$, the critical angle can be defined as Pa et al. (2008):

$$
\varphi_{c}=\sin ^{-1}\left(\frac{n_{1}}{n_{2}}\right)
$$

where $\mathrm{n}_{1}$ and $\mathrm{n}_{2}$ are refractive indices of the light-guiding (glass) and outer media (solution), respectively, when $\mathrm{n}_{2}<\mathrm{n}_{1}$. The evanescent waves extend out from the surface of the higher refractive index medium into the lower index medium. The power of the evanescent field decays exponentially. The power of the evanescent field decays exponentially with distance from the surface. The distance from the surface where the field strength is $1 / \mathrm{e}$ of its value at the surface is known as the depth of penetration $\left(d_{p}\right)$ Pa et al. (2008):

$$
d_{p}=\frac{\lambda}{2 \pi \sqrt{\left(n_{1}^{2} \sin ^{2}(\varphi)-n_{2}^{2}\right)}}
$$

The depth of penetration increases with longer wavelengths and increasing propagation angles $(\varphi)$, relative to a normal perpendicular to the capillary axis, with a maximum at $\varphi_{\max }$. So, if we assume that the evanescent wave is used to excite fluorophores, the fluorescence intensity can be described as Demello et al. (1995)

$$
I_{e}=\alpha \int_{0}^{\infty} \Phi c I_{0} e^{(-x / \Lambda)} d x
$$

where, $\mathrm{I}_{\mathrm{O}}$ is the incident light intensity, $\alpha$ is the molar absorptivity, and $\mathrm{c}$ is the concentration of the dye. The fluorescence quantum yield $(\Phi)$ is defined as the ratio of photons emitted to the photons absorbed and gives the efficiency of the fluorescence process. The quantum yield $\Phi$ of acridine orange is equal 0.2 Soep et al. (1972).

In the case of our sensor which is illuminated with the collimated beam, light enters into the solution and fluorescence is excited directly. Due to the fact that the light path in the sensor is relatively short, we can assume attenuation is negligible. Hence, intensity Id is proportional to the amount of light absorbed and the fluorescence quantum yield, $\Phi$ :

$$
I_{d}=k \Phi I_{0}\left[1-10^{(-\alpha b c)}\right]
$$

where $b$ is the path length.

Thus, the total fluorescence excited by the presented sensor can be described as a sum of direct fluorescence and the evanescent wave excited fluorescence.

In Fig. 2 the microscopic image of the part of the biosensor is presented. The biosensor is filled with the solution of the acridine orange. As one can observe, the light is propagated through the filled capillaries and the walls of the biosensor. Examples of visible fluorescence are shown by the circles. 
Fig. 2 Excitation and fluorescence signals at the distal end of the biosensor
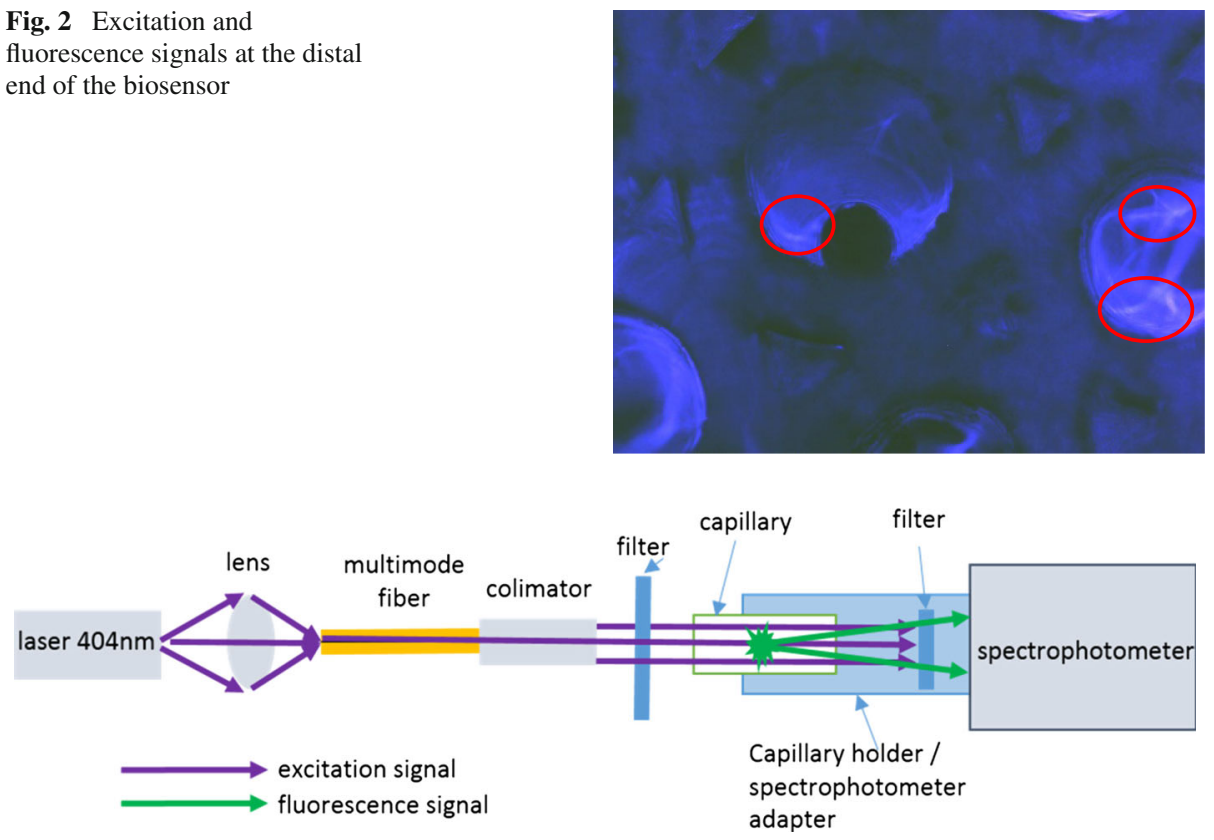

Fig. 3 Measurement setup

\section{Measurement setup}

The capillary waveguide biosensor is filled with a liquid acridine orange in a water solution and is illuminated with collimated light at wavelength $404 \mathrm{~nm}$. The multimode fiber is used in order to simplify the illumination of the capillary biosensor. The collimator is used in order to illuminate the capillaries in equal intensities. Undesirable luminescence originating from the multimode fiber is then filtered using a filter 1 . The capillary biosensor is placed in a holder which simultaneously allows the elimination of the influence of the external light and allows the use of an additional filter 2 to filter out the excitation signal (Fig. 3).

\section{Measurement results}

During the research, as a result of cutting the preform into smaller pieces, we obtained three biosensors, and we conducted 3 series of measurements for each biosensor. In order to fill the capillary in the same way, the solution was introduced into the capillary by means of a syringe. The capillary was then placed in a holder allowing almost the same input conditions of light to be maintained. Every time the solution in the capillary was changed, the capillary was washed several times in water and dried with compressed air. It is also worth mentioning that the studies have been carried out from the smallest to the largest molar concentration of the solution. Figure 4 shows the averaged measured fluorescence intensities for different molar concentrations of acridine orange solution in water as a difference between measured fluorescence from the capillary field with the solution and the capillary field with water.

Figure 5 shows the normalized average fluorescence intensities for different molar concentration of acridine orange. The error bars depict standard deviation. One can notice that 


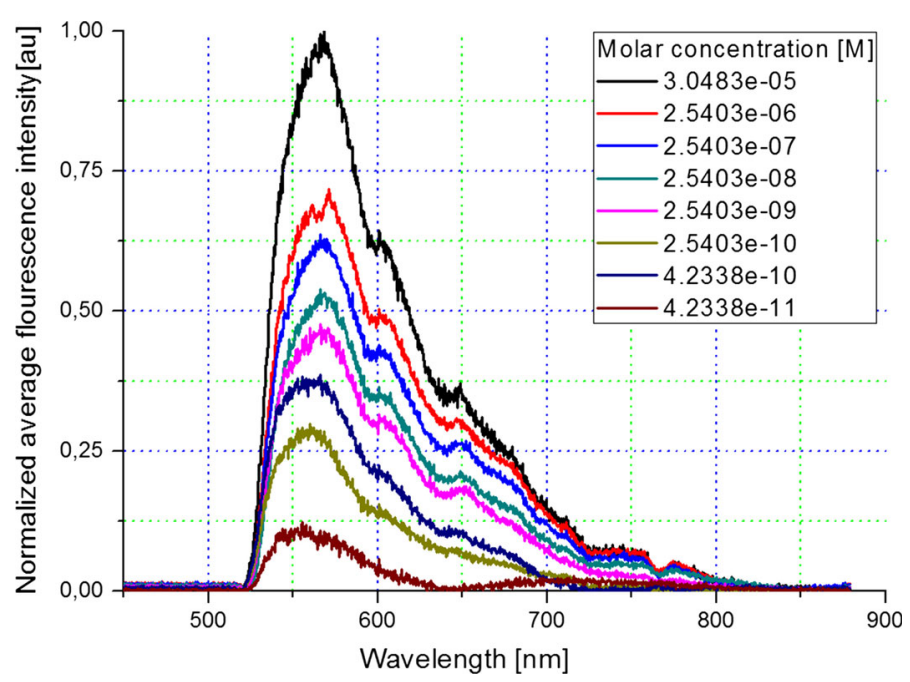

Fig. 4 Normalized average fluorescence intensity for different molar concentration of acridine orange solution in water

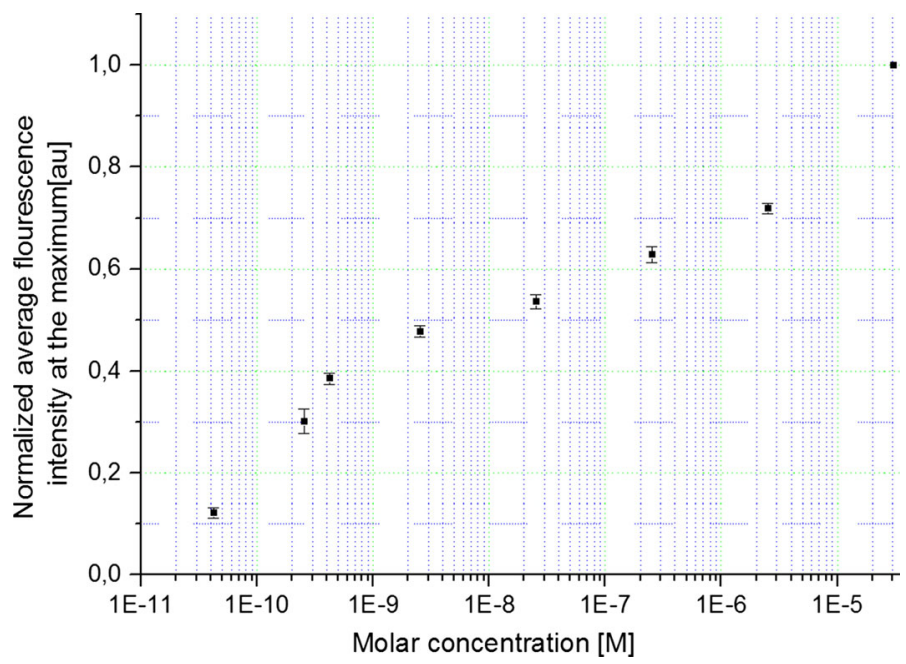

Fig. 5 Normalized fluorescence intensity at the maximum for different molar concentration of acridine orange solution in water

the florescence intensity is proportional to the concentration of dye as it was expected. As we can observe in Fig. 5 the error bars are very small which result on the repeatability of the measurement.

\section{Conclusion}

We can conclude that the proposed capillary biosensor waveguide can be used as a simple and cheap device to determine flourophores in solvent. In this approach we used acridine orange solution in water to determine the basic properties of this biosensor. 
The conducted experiment showed that although the solution was of a small volume $(\mathrm{V}=31.4 \mu \mathrm{L})$, the fluorescence signal is measured even for a very small concentration of dyes (range of $10^{-11} \mathrm{M}$ ). This is possible by using multiple short capillaries and through the excitation of fluorescence not only directly but also through an evanescent wave. Assuming that the quantum yield of NADH is twice smaller than acridine orange, the sensor presented in this approach should also allow for the detection of very small concentrations of NADH in urine. Additionally, it is possible to enhance a measured florescence signal by means of the other spectrophotometer adapter which could serve as an integrating sphere and can better detect signals from the whole biosensor.

Open Access This article is distributed under the terms of the Creative Commons Attribution License which permits any use, distribution, and reproduction in any medium, provided the original author(s) and the source are credited.

\section{References}

Rajasekaran, R., Aruna, P.R., Koteeswaran, D., Padmanabhan, L., Muthuvelu, K., Rai, R.R., Thamilkumar, P., Krishna, C.M., Ganesan, S.: Characterization and diagnosis of cancer by native fluorescence spectroscopy of human urine. Photochem. Photobiol. 89, 483-491 (2013)

Keller, B.K., DeGrandpre, M.D., Palmer, C.P.: Waveguiding properties of fiber-optic capillaries for chemical sensing applications. Sens. Actuators B 125, 360-371 (2007)

Paprocki, E.D., Keller, B.K., Palmer, C.P., Laws, W.R., DeGrandpre, M.D.: Characterization of long pathlength capillary waveguides for evanescent fluorescence sensing applications. Sens. Actuators B Chem. 135(1), 145-151 (2008)

Demello, A.J., Crystall, B., Rumbles, G.: Evanescent-wave spectroscopic studies of surface-enhanced fluorescence quantum efficiencies. J. Colloid Interface Sci. 169, 161-167 (1995)

Soep, B., Kellmann, A., Martin, M., Lindqvist, L.: Study of triplet quantum yields using a tunable dye laser. Chem. Phys. Lett. 13(3), 241-244 (1972) 